\title{
A RECURRENCE RELATED TO TREES
}

\author{
DONALD E. KNUTH AND BORIS PITTEL
}

(Communicated by William J. Davis)

\begin{abstract}
The asymptotic behavior of the solutions to an interesting class of recurrence relations, which arise in the study of trees and random graphs, is derived by making uniform estimates on the elements of a basis of the solution space. We also investigate a family of polynomials with integer coefficients, which may be called the "tree polynomials."
\end{abstract}

There are $n^{n-2}(n-1)$ ! sequences of edges between vertices

$$
u_{1}-v_{1}, \ldots, u_{n-1}-v_{n-1}, \quad 1 \leq u_{k}<v_{k} \leq n,
$$

that define a free tree on $\{1, \ldots, n\}$, because there are $n^{n-2}$ free trees on $n$ labeled vertices and every such tree has $n-1$ edges. If we consider each of these $n^{n-2}(n-1)$ ! sequences to be equally likely, the probability that $u_{n-1}$ and $v_{n-1}$ belong respectively to components of sizes $k$ and $n-k$ based on the first $n-2$ edges is

$$
p_{n k}=\frac{1}{2(n-1)}\left(\begin{array}{l}
n \\
k
\end{array}\right)\left(\frac{k}{n}\right)^{k-1}\left(\frac{n-k}{n}\right)^{n-k-1}, 0<k<n .
$$

Knuth and Schönhage $[9, \S \S 9-12]$ considered tree-construction algorithms whose analysis depended on the solution of the recurrence

$$
x_{n}=c_{n}+\sum_{0<k<n} p_{n k}\left(x_{k}+x_{n-k}\right)
$$

for various sequences $\left\langle c_{n}\right\rangle$. The purpose of the present note is to extend the results of [9] and to consider related sequences of functions whose exact and asymptotic values arise in a variety of algorithms.

Much of the analysis below, as in [9], depends on properties of the formal power series

$$
T(z)=\sum_{n \geq 1} \frac{n^{n-1} z^{n}}{n !}=z+z^{2}+\frac{3}{2} z^{3}+\frac{8}{3} z^{4}+\frac{125}{24} z^{5}+\cdots,
$$

Received by the editors March 18, 1988.

1980 Mathematics Subject Classification (1985 Revision). Primary 41A60, 12E10; Secondary 05A15, 68R99, 05C80.

This research was supported in part by the National Science Foundation under grant CCR-8610181, and by Office of Naval Research contract N00014-87-K-0502. 
which is the exponential generating function for labeled, rooted trees. $§ 1$ discusses the connection between $T(z)$ and functions that generate the sequences $\left\langle c_{n}\right\rangle,\left\langle x_{n}\right\rangle$ of $(0.3) . \S 2$ derives fundamental properties of the tree polynomials $t_{n}(y)$, defined by

$$
\frac{1}{(1-T(z))^{y}}=\sum_{n \geq 0} t_{n}(y) \frac{z^{n}}{n !}
$$

for fixed $y$ as $n \rightarrow \infty$. Asymptotic properties of the tree polynomials and related quantities are derived in $\S 3$. These results are applied in $\S 4$ to derive asymptotic properties of $\left\langle x_{n}\right\rangle$ when the asymptotic behavior of $\left\langle c_{n}\right\rangle$ is given.

We prove in particular that if $c_{n}=n^{\beta}$ and $\beta>1 / 2$ then

$$
x_{n}=\frac{\Gamma(\beta-1 / 2)}{\sqrt{2} \Gamma(\beta)} n^{\beta+1 / 2}+O\left(n^{\beta}\right)+O(n \log n) .
$$

On the other hand, if $c_{n}=O\left(n^{\beta}\right)$ and $\beta<1 / 2$ then

$$
x_{n}=n \kappa+O\left(n^{\beta+1 / 2}\right)+O(1) \text {, }
$$

where

$$
\kappa=\sum_{m \geq 1} c_{m} m^{-2}\left(1+\frac{m^{m} e^{-m}}{m !}(m-Q(m))\right)
$$

and $Q(m)$ is Ramanujan's function. Thus $x_{n}$ is superlinear in $n$ or linear in $n$ dependent upon whether $\beta>1 / 2$ or $\beta<1 / 2$.

\section{Basic SOlutions}

If $\left\langle x_{n}^{(m)}\right\rangle$ is the solution of $(0.3)$ in the special case $c_{n}=\delta_{m n}$, the general solution is

$$
x_{n}=x_{n}^{(1)} c_{1}+x_{n}^{(2)} c_{2}+\cdots+x_{n}^{(n)} c_{n}
$$

It is easy to verify that $x_{n}^{(1)}=n$ and that $x_{n}^{(1)}+x_{n}^{(2)}+\cdots=2 n-1$. Knuth and Schönhage [9] proved that

$$
x_{n}^{(2)}=\frac{n}{4}\left(1+\left(1-\frac{2}{n}\right)^{n-2}\right)
$$

and, for general $m$,

$$
\begin{aligned}
x_{n}^{(m)}=\frac{n}{m^{2}}\left(m\left(\begin{array}{c}
n \\
m
\end{array}\right)\left(1-\frac{m}{n}\right)^{n-m}\left(\frac{m}{n}\right)^{m}+1\right. \\
\left.\quad-\sum_{j=0}^{m-1}\left(\begin{array}{c}
n-1 \\
j
\end{array}\right)\left(1-\frac{m}{n}\right)^{n-1-j}\left(\frac{m}{n}\right)^{j}\right) .
\end{aligned}
$$

Notice that $x_{n}^{(m)}=0$ when $m>n$, and $x_{n}^{(n)}=1$. 
A new family of special solutions to the recurrence also proves to be important, namely

$$
x_{n}(y)=t_{n}(y) / n^{n-1}
$$

where $t_{n}(y)$ is the tree polynomial of $(0.5)$. We can find the corresponding leading terms $c_{n}(y)$ by plugging into $(0.3)$ :

$$
\frac{t_{n}(y)}{n^{n-1}}=c_{n}(y)+\frac{1}{(n-1) n^{n-2}} \sum_{k=1}^{n-1}\left(\begin{array}{l}
n \\
k
\end{array}\right) k^{k-1} t_{n-k}(y) .
$$

(We have used the fact that $p_{n k}=p_{n(n-k)} \cdot$ ). This sum is $n^{n-1}$ less than the coefficient of $z^{n} / n !$ in $T(z) /(1-T(z))^{y}=1 /(1-T(z))^{y}-1 /(1-T(z))^{y-1}$, namely $t_{n}(y)-t_{n}(y-1)$. Therefore we have

$$
c_{n}(y)=\frac{n t_{n}(y-1)-t_{n}(y)+n^{n}}{(n-1) n^{n-1}}, \quad \text { for } n \geq 2 ;
$$

and $c_{1}(y)=t_{1}(y)=y$.

The value of $c_{n}(y)$ can also be expressed in another form, because of an interesting identity satisfied by the tree polynomials. The well-known equation

$$
T(z)=z e^{T(z)},
$$

due to Eisenstein [1], implies that

$$
T^{\prime}(z)=\frac{T(z)}{z(1-T(z))} .
$$

Therefore

$$
\sum_{n \geq 0} \frac{n t_{n}(y-1) z^{n}}{n !}=z \frac{d}{d z} \frac{1}{(1-T(z))^{y-1}}=\frac{(y-1) T(z)}{(1-T(z))^{y+1}}
$$

and we have the difference equation

$$
t_{n}(y+1)=t_{n}(y)+n \frac{t_{n}(y-1)}{y-1} .
$$

It follows that

$$
c_{n}(y)=\frac{(y-1) t_{n}(y+1)-y t_{n}(y)+n^{n}}{(n-1) n^{n-1}} .
$$

\section{TREE POLYNOMIALS}

The first few cases of the polynomials $t_{n}(y)$ defined in (0.5) are

$$
\begin{aligned}
& t_{0}(y)=1, \\
& t_{1}(y)=y, \\
& t_{2}(y)=y^{2}+3 y, \\
& t_{3}(y)=y^{3}+9 y^{2}+17 y, \\
& t_{4}(y)=y^{4}+18 y^{3}+95 y^{2}+142 y .
\end{aligned}
$$


Our derivation of (1.6) from (1.5) was, in essence, a proof of the difference equation

$$
t_{n}(y)-t_{n}(y-1)=\sum_{k=1}^{n}\left(\begin{array}{l}
n \\
k
\end{array}\right) k^{k-1} t_{n-k}(y) .
$$

Therefore, by (1.9), we have

$$
t_{n}(y-2)=\frac{y-2}{n} \sum_{k=1}^{n}\left(\begin{array}{l}
n \\
k
\end{array}\right) k^{k-1} t_{n-k}(y),
$$

a recurrence by which $t_{n}(y)$ can be obtained when $t_{n-1}(y), \ldots, t_{0}(y)$ are known. Two analogous formulas can also be proved:

$$
\begin{aligned}
& t_{n}(y+1)=\sum_{k=0}^{n}\left(\begin{array}{l}
n \\
k
\end{array}\right) k^{k} t_{n-k}(y) \\
& t_{n}^{\prime}(y)=\sum_{k=1}^{n}\left(\begin{array}{l}
n \\
k
\end{array}\right) k^{k-1} Q(k) t_{n-k}(y) .
\end{aligned}
$$

Here $Q(n)$ is Ramanujan's function

$$
\begin{aligned}
Q(n) & =1+\frac{n-1}{n}+\frac{n-1}{n} \frac{n-2}{n}+\frac{n-1}{n} \frac{n-2}{n} \frac{n-3}{n}+\cdots \\
& =\sqrt{\frac{\pi n}{2}}-\frac{1}{3}+\frac{1}{12} \sqrt{\frac{\pi}{2 n}}-\frac{4}{135 n}+O\left(n^{-3 / 2}\right),
\end{aligned}
$$

which arises in the analysis of many algorithms (see [5]). Equation (2.4) follows from the well-known identity (Riordan [12])

$$
\frac{1}{1-T(z)}=\sum_{n \geq 0} \frac{n^{n} z^{n}}{n !}
$$

which gives the exponential generating function for $n^{n}$, the number of all mappings of the set $\{1, \ldots, n\}$ into itself. Equation (2.5) follows from

$$
\ln \frac{1}{1-T(z)}=\sum_{n \geq 1} \frac{C(n) z^{n}}{n !}
$$

where $C(n)$ is the total number of all one-cycle mappings [12], and from a formula

$$
C(n)=n^{n-1} Q(n),
$$

which is due to Rényi [11]. In general, we have the formal relation

$$
\begin{aligned}
& a_{1} T(z)+a_{2} T(z)^{2}+a_{3} T(z)^{3}+\cdots \\
& \quad=\sum_{n \geq 1} \frac{n^{n-1} z^{n}}{n !}\left(a_{1}+2 a_{2} \frac{n-1}{n}+3 a_{3} \frac{n-1}{n} \frac{n-2}{n}+\cdots\right),
\end{aligned}
$$

by Lagrange's inversion formula; equations (2.7) and (2.8) are special cases of this identity. 
Equation (2.4) is also a special case of the convolution formula

$$
t_{n}\left(y_{1}+y_{2}\right)=\sum_{k=0}^{n}\left(\begin{array}{l}
n \\
k
\end{array}\right) t_{k}\left(y_{1}\right) t_{n-k}\left(y_{2}\right) .
$$

If $F(z)$ is any formal power series such that $\ln F(z)=\sum_{n \geq 1} b_{n} z^{n} / n !$, the coefficient of $y^{m} z^{n} / n$ ! in $F(z)^{y}=e^{y \ln F(z)}$ is a multinomial convolution

$$
\begin{aligned}
& \frac{1}{m !} \sum_{\substack{k_{1}+\cdots+k_{m}=n \\
k_{1}, \ldots, k_{m}>0}}\left(\begin{array}{c}
n \\
k_{1}, \ldots, k_{m}
\end{array}\right) b_{k_{1}} \cdots b_{k_{m}} \\
& =\sum_{\substack{r_{1}+r_{2}+\cdots=m \\
r_{1}+2, r_{2}+\cdots=n \\
r_{1}, r_{2}, \ldots \geq 0}} \frac{n !}{1 !^{r_{1}} r_{1} ! 2 !^{r_{2}} r_{2} ! \cdots} b_{1}^{r_{1}} b_{2}^{r_{2}} \cdots .
\end{aligned}
$$

(This is essentially Faà di Bruno's formula [6, exercise 1.2.5-21].) In our case each $b_{k}=C(k)$ is an integer, so the coefficients of the tree polynomials $t_{n}(y)$ are integers.

Indeed, the coefficients of $t_{n}(y)$ have a natural combinatorial interpretation, as noted by Flajolet and Soria [4, Example 4]: The coefficient of $y^{k}$ is the number of mappings of $\{1,2, \ldots, n\}$ into itself such that the corresponding functional digraph has exactly $k$ components. (Equivalently, the mapping has exactly $k$ different cycles; see [7, exercise 3.1-14].) The reason is simply that the corresponding exponential generating function, according to (2.8) and well-known techniques for enumerating labeled configurations, is $\exp (y \ln (1 /(1-T(z))))$, and this is just (0.5). Stepanov [13] showed that these coefficients are asymptotically normal with mean and variance $\frac{1}{2} \ln n+O(1)$; Flajolet and Soria [4] extended this to a general result on the number of components in random labeled structures.

We wish to study the asymptotic behavior of $t_{n}(y)$ for fixed $y$ as $n \rightarrow \infty$, and for this we need another formula based on (2.9): The binomial expansion $(1-T(z))^{-y}=\sum_{k \geq 0}\left(\begin{array}{c}y+k-1 \\ k\end{array}\right) T(z)^{k}$ and equation (2.9) imply that

$$
\begin{aligned}
t_{n}(y) & =n^{n-1}\left(\frac{y}{0 !}+\frac{y(y+1)}{1 !} \frac{n-1}{n}+\frac{y(y+1)(y+2)}{2 !} \frac{n-1}{n} \frac{n-2}{n}+\cdots\right) \\
& =\frac{n^{n-1}}{\Gamma(y)} \sum_{k \geq 0}\left(\begin{array}{c}
n-1 \\
k
\end{array}\right) \frac{\Gamma(y+k+1)}{n^{k}} .
\end{aligned}
$$

Integral formulas for $t_{n}(y)$ are also of interest:

$$
\begin{aligned}
t_{n}(y) & =n^{n} y \int_{0}^{\infty} \sum_{k}\left(\begin{array}{c}
n-1 \\
k
\end{array}\right)\left(\begin{array}{c}
y+k \\
k
\end{array}\right) t^{k} e^{-n t} d t \\
& =n^{n}+n^{n+1} \int_{0}^{\infty} \sum_{k}\left(\begin{array}{c}
n-1 \\
k
\end{array}\right)\left(\begin{array}{c}
y+k-1 \\
k+1
\end{array}\right) t^{k} e^{-n t} d t .
\end{aligned}
$$




\section{ASYMPTOTIC LeMMAS}

The terms of (2.12) increase until $k$ is approximately $\sqrt{y n}$, after which they begin to decrease rather rapidly. More explicitly, when $y$ and $\varepsilon>0$ are fixed and $0<k \leq n^{1 / 2+\varepsilon}$, we have

$$
\begin{aligned}
& \frac{\Gamma(y+k+1)}{k !}=k^{y}\left(1+\frac{y^{2}+y}{2 k}+O\left(k^{-2}\right)\right), \\
& \frac{(n-1) !}{(n-1-k) ! n^{k}}=e^{-k^{2} / 2 n}\left(1-\frac{k}{2 n}-\frac{k^{3}}{6 n^{2}}+O\left(n^{-1+6 \varepsilon}\right)\right),
\end{aligned}
$$

by Stirling's approximation. Therefore the terms of (2.12) are exponentially small when $k \geq n^{1 / 2+\varepsilon}$, and we can replace them with other exponentially small terms to get

$$
\begin{aligned}
t_{n}(y)=\frac{n^{n-1}}{\Gamma(y)}( & S_{2 n}(y)+\frac{y^{2}+y}{2} S_{2 n}(y-1) \\
& \left.-\frac{1}{2 n} S_{2 n}(y+1)-\frac{1}{6 n^{2}} S_{2 n}(y+3)+R_{n}(y)\right)
\end{aligned}
$$

where

$$
S_{n}(y)=\sum_{k \geq 1} k^{y} e^{-k^{2} / n}
$$

and where $R_{n}(y)$ is an error term to be determined below.

When $y$ is a nonnegative integer, the sum $S_{n}(y)$ is essentially a theta function whose asymptotic behavior is well known. When $y=-1$, the analysis of bubble sort in Knuth [8, exercise 5.2.2-4] shows that $S_{n}(-1)=\frac{1}{2} \ln n+\frac{1}{2} \gamma+$ $O\left(n^{-1}\right)$. For our purposes we want to consider $S_{n}(y)$ when $y$ is a real number larger than -1 . It turns out that $S_{n}(y)=\int_{0}^{\infty} x^{y} e^{-x^{2} / n} d x+O(1)$ in this case, and we can continue the asymptotic series explicitly:

Lemma 1. If $y>-1$, the series $S_{n}(y)$ defined in (3.4) satisfies

$$
S_{n}(y)=\frac{1}{2} \Gamma\left(\frac{y+1}{2}\right) n^{(y+1) / 2}+\sum_{j=0}^{m} \frac{(-1)^{j} \zeta(-y-2 j)}{j ! n^{j}}+O\left(n^{-m-1}\right),
$$

for all $m \geq 0$, as $n \rightarrow \infty$.

Proof. Consider the related sum

$$
\begin{aligned}
\bar{S}_{n, N} & =\sum_{k=1}^{N \sqrt{n}-1} k^{y}\left(e^{-k^{2} / n}-\frac{1}{0 !}+\frac{k^{2}}{1 ! n}-\cdots+\frac{(-1)^{m+1} k^{2 m}}{m ! n^{m}}\right) \\
& =n^{y / 2} \sum_{k=1}^{N \sqrt{n}-1} g_{m}\left(\frac{k}{\sqrt{n}}\right),
\end{aligned}
$$


where

$$
g_{m}(x)=x^{y}\left(e^{-x^{2}}-\sum_{j=0}^{m} \frac{\left(-x^{2}\right)^{j}}{j !}\right)
$$

Euler's summation formula tells us that

$$
\bar{S}_{n, N}=n^{(y+1) / 2} \int_{0}^{N} g_{m}(x) d x+\sum_{l=1}^{p} \frac{B_{l}}{l !} g_{m}^{(l-1)}(N) n^{(y+1-l) / 2}+R_{p},
$$

where

$$
R_{p}=\frac{(-1)^{p+1}}{p !} n^{(y+1-p) / 2} \int_{0}^{N} B_{p}(\{x / \sqrt{n}\}) g_{m}^{(p)}(x) d x,
$$

if $p$ is chosen small enough that $\lim _{x \rightarrow 0} g_{m}^{(p-1)}(x)=0$. The latter condition is satisfied if $p<y+2 m+3$, since $g_{m}(x)=O\left(x^{y+2 m+2}\right)$ as $x \rightarrow 0$. If we choose $p$ so that $y+2 m+1<p<y+2 m+3$, we can in fact prove that the integral $\int_{0}^{\infty}\left|g_{m}^{(p)}(x)\right| d x$ is finite, because $g_{m}^{(p)}(x)$ is $O\left(x^{y+2 m+2-p}\right)$ as $x \rightarrow 0$ and its terms that do not involve $e^{-x^{2}}$ are $O\left(x^{y+2 m-p}\right)$ as $x \rightarrow \infty$. Therefore $R_{p}=O\left(n^{(y+1-p) / 2}\right)=O\left(n^{-m}\right)$ for this choice of $p$, uniformly in $N$.

Euler's summation formula also implies the identity

$$
\sum_{k=1}^{M-1} k^{\alpha}=\zeta(-\alpha)+\sum_{l=0}^{p}\left(\begin{array}{c}
\alpha+1 \\
l
\end{array}\right) B_{l} \frac{M^{\alpha+1-l}}{\alpha+1}+O\left(M^{\alpha-p}\right) ;
$$

indeed, this is the traditional way to define the zeta function. Therefore if

$$
h_{m}(x)=x^{y} \sum_{j=0}^{m} \frac{\left(-x^{2}\right)^{j}}{j !}
$$

is the "smoothing function" included in $g_{m}(x)$, we have

$$
\begin{aligned}
n^{y / 2} \sum_{k=1}^{N \sqrt{n}-1} h_{m}\left(\frac{k}{\sqrt{n}}\right)= & n^{(y+1) / 2} \int_{0}^{N} h_{m}(x) d x+\sum_{j=0}^{m} \frac{(-1)^{j} \zeta(-y-2 j)}{j ! n^{j}} \\
& +\sum_{l=1}^{p} \frac{B_{l}}{l !} h_{m}^{(l-1)}(N) n^{(y+1-l) / 2}+O\left(n^{-m}\right) .
\end{aligned}
$$

Adding (3.8) to (3.12) and letting $N \rightarrow \infty$ completes the proof of (3.5), except that the error estimate is $O\left(n^{-m}\right)$ instead of $O\left(n^{-m-1}\right)$. To obtain the sharper estimate, we simply replace $m$ by $m+1$.

Lemma 2. The tree polynomial $t_{n}(y)$ defined in (0.5) satisfies

$$
t_{n}(y)=\frac{\sqrt{2 \pi} n^{n-1}}{2^{y / 2}}\left(\frac{n^{(y+1) / 2}}{\Gamma(y / 2)}+\frac{\sqrt{2} y}{3} \frac{n^{y / 2}}{\Gamma((y-1) / 2)}+O\left(n^{(y-1) / 2}\right)+O(1)\right),
$$

for fixed $y$ as $n \rightarrow \infty$. 
Proof. We may assume that $y>-1$, for if $y=-1$ the stated result is consistent with the fact that $t_{n}(-1)=-n^{n-1}$ and if $y<-1$ we easily have $t_{n}(y)=O\left(n^{n-1}\right)$.

The contribution to the error term $R_{n}(y)$ in (3.3) that arises from $O\left(n^{-1+6 \varepsilon}\right)$ in (3.2) is $O\left(n^{(y-1) / 2+6 \varepsilon}\right)$, by Lemma 1; this can be improved to $O\left(n^{(y-1) / 2}\right)$ by carrying out the expansion in (3.2) a bit further. The contribution to $R_{n}(y)$ from $O\left(k^{-2}\right)$ in (3.1) is $O\left(n^{(y-1 / 2)}\right)$ if $y>1$. If $y=1$, the $O\left(k^{-2}\right)$ of (3.1) is actually zero; and if $y<1$ the contribution to $R_{n}(y)$ is $O(1)$ because $S_{n}(y-2)<\sum_{k \geq 1} k^{y-2}$.

To complete the estimation of $t_{n}(y)$ from (3.3), we need only the weak form $S_{n}(y)=\frac{1}{2} \Gamma((y+1) / 2) n^{(y+1) / 2}+O(1)$ of Lemma 1. Application of the duplication formula $\Gamma(y)=2^{y-1} \Gamma(y / 2) \Gamma((y+1) / 2) / \sqrt{\pi}$ completes the proof.

Lemma 2 can be verified, for example, in the special cases

$$
t_{n}(1)=n^{n} ; \quad t_{n}(2)=n^{n}(Q(n)+1) .
$$

Computer calculations show that the series in Lemma 2 continues as follows:

$$
\begin{aligned}
t_{n}(y)=n^{n-1}( & f_{n}(y)+\frac{y}{3} f_{n}(y-1)+\frac{(y-1)(y+1)(2 y-3)}{36(y-2)} f_{n}(y-2) \\
& \left.+\frac{y(y+2)\left(10 y^{2}-35 y+24\right)}{1620(y-3)} f_{n}(y-3)+O\left(n^{(y-3) / 2}\right)+O(1)\right),
\end{aligned}
$$

where $f_{n}(y)=\sqrt{2 \pi} n^{(y+1) / 2} /\left(2^{y / 2} \Gamma(y / 2)\right)$. Stronger methods than those above will be needed to penetrate the $O(1)$ term.

Philippe Flajolet has pointed out, in a personal communication to the authors, that Lemma 2 can also be deduced directly from a singularity analysis as in [3]. Indeed, we have

$$
T(z)=1-S(\sqrt{2-2 e z}),
$$

where the analytic function

$$
S(z)=z-\frac{1}{3} z^{2}+\frac{11}{72} z^{3}-\frac{43}{540} z^{4}+\frac{769}{17280} z^{5}-\frac{221}{8505} z^{6}+\cdots
$$

satisfies the functional relation

$$
(1-S(z)) e^{S(z)}=1-\frac{z^{2}}{2}
$$

and has a Maclaurin series that converges for $|z|<\sqrt{2}$. This leads to an asymptotic expansion of the coefficients of $(1-T(z))^{-y}=S(\sqrt{2-2 e z})^{-y}$. And in fact, the resulting expansion holds for all $y$; we can actually eliminate the term $O(1)$ from (3.13) and (3.15). (We do not, however, require this strengthening of Lemma 2 in the calculations below.) 
It is interesting to observe that (1.9) implies

$$
t_{n}(y)=n\left(\frac{t_{n}(y-2)}{y-2}+\frac{t_{n}(y-3)}{y-3}+\cdots+\frac{t_{n}(y-m)}{y-m}\right)+t_{n}(y-m+1)
$$

the terms on the right decrease by factors of order $\sqrt{n}$ until order $n^{n-1}$ is reached.

Lemma 3. If $y>0$, we have

$$
\frac{t_{n}^{\prime}(y)}{t_{n}(y)}=\frac{1}{2}\left(\ln \frac{n}{2}+\gamma-H_{y / 2-1}\right)+O\left(n^{-1 / 2}\right)
$$

where

$$
H_{y}=\sum_{k \geq 1}\left(\frac{1}{k}-\frac{1}{k+y}\right) .
$$

Proof. The calculations in the proofs of Lemmas 1 and 2 also support differentiation by $y$. The right-hand side of $(3.20)$ is $f_{n}^{\prime}(y) / f_{n}(y)$, because of the well-known relation

$$
H_{y}=\frac{\Gamma^{\prime}(y+1)}{\Gamma(y+1)}+\gamma .
$$

(See [6, exercise 1.2.7-23].)

The special case $y=1$ of Lemma 3 is of particular interest. According to (0.5), (2.7), and (2.8), we have

$$
\begin{aligned}
t_{n}^{\prime}(1) & =n !\left[z^{n}\right] \frac{1}{1-T(z)} \ln \frac{1}{1-T(z)} \\
& =\sum_{k=1}^{n}\left(\begin{array}{l}
n \\
k
\end{array}\right) k^{k-1} Q(k)(n-k)^{n-k} .
\end{aligned}
$$

By our combinatorial interpretation of $t_{n}(y)$, this is the total number of cycles among all mappings of $\{1,2, \ldots, n\}$ into itself. Lemma 3 and equation (3.14) imply that

$$
t_{n}^{\prime}(1)=\frac{1}{2} n^{n}\left(\ln n+\gamma+\ln 2+O\left(n^{-1 / 2}\right)\right) \text {, }
$$

a formula first obtained by Martin Kruskal [10]. The $O\left(n^{-1 / 2}\right)$ can be refined further to $\frac{1}{6} \sqrt{2 \pi / n}+O\left(n^{-1} \log n\right)$. In fact, the general formula (3.15) can be differentiated with respect to $y$; the $O(1)$ remains $O(1)$.

The derivative of the formula in Lemma 1 with respect to $y$ is sufficiently interesting to be stated explicitly. We have

$$
\begin{aligned}
\sum_{k=1}^{\infty} k^{y} \ln k e^{-k^{2} / n}= & \frac{1}{4} \Gamma\left(\frac{y+1}{2}\right) n^{(y+1) / 2}\left(\ln n+\gamma+H_{(y-1) / 2}\right) \\
& +\sum_{j=0}^{m} \frac{(-1)^{j} \zeta^{\prime}(-y-2 j)}{j ! n^{j}}+O\left(n^{-m-1}\right),
\end{aligned}
$$

for all $y>-1$. 
We will also need asymptotic estimates of the basic functions $x_{n}^{(m)}$ of (1.3), valid for all $m$ and $n$.

Lemma 4. Let

$$
\alpha_{m}=\frac{1}{m^{2}}\left(1+\frac{m^{m} e^{-m}}{m !}(m-Q(m))\right),
$$

where $Q(m)$ is Ramanujan's function (see (2.6)). The quantity $x_{n}^{(m)}$ defined in (1.3) satisfies

$$
x_{n}^{(m)}=n \alpha_{m}+O\left(m^{-1 / 2}\right)+O\left((n-m)^{-1 / 2}\right),
$$

uniformly for $1 \leq m \leq n-1$.

Proof. With Stirling's approximation, we can rewrite the main term of (1.3) as follows:

$$
\begin{aligned}
\left(\begin{array}{c}
n \\
m
\end{array}\right)\left(1-\frac{m}{n}\right)^{n-m}\left(\frac{m}{n}\right)^{m} & =\frac{n !}{n^{n} e^{-n}} \frac{m^{m} e^{-m}}{m !} \frac{(n-m)^{n-m} e^{-(n-m)}}{(n-m) !} \\
& =\frac{m^{m} e^{-m}}{m !} \sqrt{\frac{n}{n-m}}\left(1+O\left(\frac{1}{n-m}\right)\right)
\end{aligned}
$$

Since

$$
\sqrt{\frac{n}{n-m}}=1+\frac{m}{\sqrt{n-m}(\sqrt{n}+\sqrt{n-m})},
$$

the right-hand side of (3.28) is

$$
\frac{m^{m} e^{-m}}{m !}\left(1+O\left(\frac{1}{n-m}\right)+O\left(\frac{m}{\sqrt{n(n-m)}}\right)\right) \text {. }
$$

The other terms of (1.3) are

$$
\begin{aligned}
& \left(\begin{array}{c}
n-1 \\
j
\end{array}\right)\left(1-\frac{m}{n}\right)^{n-1-j}\left(\frac{m}{n}\right)^{j} \\
& \quad=\frac{m^{j}}{j !} \frac{n !}{n^{n} e^{-n}} \frac{(n-1-j)^{n-1-j}}{(n-1-j) !} \frac{e^{j+1-n}}{e^{j+1}}\left(\frac{n-m}{n-1-j}\right)^{n-1-j} .
\end{aligned}
$$

If $m \leq n^{1 / 2}$ we have

$$
\left(\frac{n-m}{n-1-j}\right)^{n-1-j}=e^{j+1-m}\left(1+O\left(\frac{(m-1-j)^{2}}{n}\right)\right)
$$

so the entire term is

$$
\frac{m^{j} e^{-m}}{j !}\left(1+O\left(\frac{(m-1-j)^{2}}{n}\right)\right)
$$

uniformly in $j$ and $m$. Summing over $j$ gives

$$
\sum_{j=0}^{m-1}\left(\begin{array}{c}
n-1 \\
j
\end{array}\right)\left(1-\frac{m}{n}\right)^{n-1-j}\left(\frac{m}{n}\right)^{j}=\frac{m^{m} e^{-m}}{m !} Q(m)\left(1+O\left(\frac{m}{n}\right)\right)
$$


because $\sum_{j=0}^{m-1} m^{j} / j !=m^{m} Q(m) / m !$ and

$$
\begin{aligned}
\sum_{j=0}^{m-1} \frac{m^{j}}{j !}(m-j-1)^{2} & =\sum_{j=0}^{m-1} \frac{m^{j}}{j !}\left((m-1)^{2}-(2 m-3) j+j(j-1)\right) \\
& =\frac{m^{m}}{m !}((m+1) Q(m)-2 m) .
\end{aligned}
$$

Hence, for $m \leq n^{1 / 2}$ we have (see (3.28), (3.29))

$$
\begin{aligned}
x_{n}^{(m)} & =\frac{n}{m^{2}}\left(m \frac{m^{m} e^{-m}}{m !}\left(1+O\left(\frac{m}{n}\right)\right)+1-\frac{m^{m} e^{-m}}{m !} Q(m)\left(1+O\left(\frac{m}{n}\right)\right)\right) \\
& =n \alpha_{m}+O\left(m^{-1 / 2}\right) .
\end{aligned}
$$

We now consider the case $m>n^{1 / 2}$. The sum in (3.30) is the probability $P_{n m}$ that the total number of successes in $n-1$ Bernoulli trials does not exceed $m-1$, where the probability of success in each trial equals $p=m / n$. By the Berry-Esséen theorem (Feller [2]), we can write then

$$
P_{n m}=\Phi\left(-\left(\frac{n-m}{m(n-1)}\right)^{1 / 2}\right)+O\left(\frac{\rho}{\sigma^{3} \sqrt{n}}\right)
$$

here $\Phi(\cdot)$ is the standard Gaussian distribution, and

$$
\begin{gathered}
\sigma^{2}=p q=\frac{m}{n}\left(1-\frac{m}{n}\right), \\
\rho=p q\left(p^{2}+q^{2}\right) \leq p q .
\end{gathered}
$$

Since $\Phi(0)=1 / 2$, the relation (3.31) leads to

$$
P_{n m}=1 / 2+O\left((m(1-m / n))^{-1 / 2}\right) .
$$

Applying the same theorem to the Poisson distribution, or using (2.6), we also obtain

$$
\sum_{j=0}^{m-1} \frac{m^{j} e^{-m}}{j !}=1 / 2+O\left(m^{-1 / 2}\right) .
$$

According to (1.3), (3.29), (3.32), and (3.33), we can write then for $m>n^{1 / 2}$,

$$
\begin{aligned}
x_{n}^{(m)}= & \frac{n}{m^{2}}\left(m \frac{m^{m} e^{-m}}{m !}\left(1+O\left(\frac{m}{n^{1 / 2}(n-m)^{1 / 2}}\right)\right)+1\right. \\
& \left.\quad-\sum_{j=0}^{m-1} \frac{m^{j} e^{-m}}{j !}+O\left(\frac{n^{1 / 2}}{m^{1 / 2}(n-m)^{1 / 2}}\right)\right) \\
= & n \alpha_{m}+O\left(\frac{n^{1 / 2}}{m^{1 / 2}(n-m)^{1 / 2}}\right) .
\end{aligned}
$$

By considering two subcases $m<n / 2$ and $n / 2 \leq m<n$ separately, we immediately get (3.27). 
The proof of Lemma 4 is now complete.

\section{THE RECURRENCE WITH POLYNOMIAL GROWTH}

We are now ready to return to the recurrence (0.3), and to derive the asymptotic behavior of $x_{n}$ when $c_{n}$ is a power of $n$.

Theorem 1. Let $c_{n}=n^{\beta}$ for all $n>0$, and let

$$
l(\beta)=\frac{\Gamma(\beta-1 / 2)}{\sqrt{2} \Gamma(\beta)} .
$$

Then the solution to (0.3) satisfies

$$
x_{n}= \begin{cases}l(\beta) n^{\beta+1 / 2}+O\left(n^{\beta}\right), & \text { if } \beta>1 ; \\ l(\beta) n^{3 / 2}+O(n \log n), & \text { if } \beta=1 ; \\ l(\beta) n^{\beta+1 / 2}+O(n), & \text { if } \frac{1}{2}<\beta<1 ; \\ (2 \pi)^{-1 / 2} n \ln n+O(n), & \text { if } \beta=\frac{1}{2} ; \\ O(n), & \text { if } \beta<\frac{1}{2} .\end{cases}
$$

Proof. The special cases $\beta=1$ and $\beta=\frac{1}{2}$ have already been considered by Knuth and Schönhage [9, (12.7) and (12.9)], who showed in fact that the recurrence with $c_{n}=n$ has a closed form solution

$$
x_{n}=(n-1) Q(n)+n K(n) \text {. }
$$

Here $K(n)$ is Kruskal's function from (3.24),

$$
K(n)=\frac{t_{n}^{\prime}(1)}{t_{n}(1)}=1+\frac{1}{2} \frac{n-1}{n}+\frac{1}{3} \frac{n-1}{n} \frac{n-2}{n}+\cdots .
$$

Another closed form that works when $\beta=\frac{1}{2}$ is the pair of sequences

$$
c_{n}=\frac{n}{n-1}(Q(n)-1), \quad x_{n}=n K(n)-Q(n), \quad n \geq 2 ;
$$

in this case we let $c_{1}=x_{1}=0$.

For other values of $\beta$ we use the sequences $c_{n}(y)$ and $x_{n}(y)$ of (1.5) and (1.10). Lemma 2 tells us that

$$
\begin{aligned}
c_{n}(y) & =\frac{(y-1) t_{n}(y+1)-y t_{n}(y)+n^{n}}{(n-1) n^{n-1}} \\
& =\frac{\sqrt{4 \pi} n^{y / 2}}{2^{y / 2} \Gamma((y-1) / 2)}+O\left(n^{(y-1) / 2}\right)+1+O\left(n^{-1}\right) ; \\
x_{n} & =\frac{t_{n}(y)}{n^{n-1}}=\frac{\sqrt{2 \pi}}{2^{y / 2}} \frac{n^{(y+1) / 2}}{\Gamma(y / 2)}+O\left(n^{y / 2}\right)+O(1) .
\end{aligned}
$$

Let $y=2 \beta$. If $\beta \leq 0$, so that $c_{n}=n^{\beta}=O(1)$, we clearly have $x_{n}=O(n)$, hence we may assume that $\beta>0$. Therefore $y>0$, and the terms $O\left(n^{-1}\right)$ and $O(1)$ in (4.6) and (4.7) are irrelevant for our present purposes. 
The special sequence $c_{n}=n^{\beta}$ is equal to $2^{y / 2} \Gamma((y-1) / 2) c_{n}(y) / \sqrt{4 \pi}+c_{n}^{\prime}$, where $c_{n}^{\prime}=1+O\left(n^{\beta-1 / 2}\right)$. Thus $x_{n}=2^{y / 2} \Gamma((y-1) / 2) x_{n}(y) / \sqrt{4 \pi}+x_{n}^{\prime}=$ $l(\beta) n^{\beta+1 / 2}+x_{n}^{\prime}+O\left(n^{\beta}\right)$. If $0<\beta<1 / 2$, we have $x_{n}=O(n)$ because $x_{n}^{\prime}=$ $O(n)$. If $\beta>1 / 2$, the estimates in (4.2) for $\beta>\frac{1}{2}$ now follow by induction on the integer $\lfloor 2 \beta-1\rfloor$.

The asymptotic growth of $x_{n}$ when $c_{n}=n^{\beta} \ln n$ can be obtained by differentiating the results of Theorem 1 with respect to $\beta$.

To complete our study, we would like to investigate the case $\beta<1 / 2$ in greater detail, by showing that $x_{n} / n$ often approaches a constant $\kappa$ (depending on $\left.\left\langle c_{n}\right\rangle\right)$, and by estimating the difference $x_{n}-\kappa n$ for large $n$. define

If $\left\langle c_{n}\right\rangle$ is any sequence such that $\sum_{m \geq 1} c_{m} / m^{3 / 2}$ converges absolutely, let us

$$
\kappa\left\langle c_{n}\right\rangle=\sum_{m \geq 1} c_{m} \alpha_{m},
$$

where $\alpha_{m}$ is the quantity defined in Lemma 4, equation (3.26).

Theorem 2. If $\beta<\frac{1}{2}$ and $c_{n}=O\left(n^{\beta}\right)$, the solution $x_{n}$ to (0.3) satisfies

$$
x_{n}=n \kappa\left\langle c_{n}\right\rangle+O\left(n^{\beta+1 / 2}\right) \text {. }
$$

Proof. We have $x_{n}=\sum_{m \geq 1} x_{n}^{(m)} c_{m}$, so we need to apply the estimates of Lemma 4 to the sum $\sum_{m \geq 1} \varepsilon_{n}^{(m)} c_{m}$ where

$$
\varepsilon_{n}^{(m)}=x_{n}^{(m)}-n \alpha_{m} .
$$

The sum splits into four parts, of which the first and second are sufficiently small because of (3.27):

$$
\begin{aligned}
\sum_{m=1}^{n / 2} \varepsilon_{n}^{(m)} c_{m} & =\sum_{m=1}^{n / 2} O\left(m^{\beta-1 / 2}\right)=O\left(n^{\beta+1 / 2}\right) \\
\sum_{m=n / 2}^{n-1} \varepsilon_{n}^{(m)} c_{m} & =\sum_{m=n / 2}^{n-1} O\left((n-m)^{-1 / 2} n^{\beta}\right) \\
& =\sum_{m=1}^{n / 2} O\left(m^{1 / 2} n^{\beta}\right)=O\left(n^{\beta+1 / 2}\right) \\
\varepsilon_{n}^{(n)} c_{n} & =\left(1-n \alpha_{n}\right) c_{n}=O\left(n^{\beta}\right) \\
\sum_{m>n} \varepsilon_{n}^{(m)} c_{m} & =\sum_{m>n}(-n) \alpha_{m} c_{m}=O\left(n^{\beta+1 / 2}\right)
\end{aligned}
$$

The quantity $\kappa\left\langle c_{n}\right\rangle$ can, incidentally, be written as an integral instead of as a sum:

$$
\kappa\left\langle c_{n}\right\rangle=c_{1}+\int_{0}^{e^{-1}} \frac{c(w) d w}{w T(w)}=c_{1}+\int_{0}^{1} u^{-2} C\left(u e^{-u}\right)(1-u) d u
$$


where

$$
C(z)=\sum_{n \geq 2} \frac{(n-1) n^{n-1} c_{n}}{n !} z^{n}
$$

Some sort of smoothness condition is necessary for the validity of (4.9); we cannot conclude that $x_{n} / n$ approaches a limit if we know only that $\sum_{m \geq 1} c_{m} / m^{3 / 2}$ exists. For example, we might have

$$
c_{n}= \begin{cases}0, & \text { if } n \text { is not a power of } 2 ; \\ 3^{k} / k^{2}, & \text { if } n=2^{k} .\end{cases}
$$

Then $x_{n}=n^{3 / 2} /(\lg n)^{2}+O(n)$ when $n=2^{k}$, but $x_{n}=O(n)$ when $n=2^{k}-1$.

It is, however, possible to prove that

$$
\liminf _{n \rightarrow \infty} \frac{x_{n}}{n}=\kappa\left\langle c_{n}\right\rangle,
$$

if $\sum_{m \geq 1} c_{m} / m^{3 / 2}$ exists and if all $c_{n}$ are nonnegative. For in this case we can define $c_{n}^{\prime}=c_{n}$ for $n \leq N$ and $c_{n}^{\prime}=0$ for $n>N$; then $x_{n} / n \geq x_{n}^{\prime} / n \rightarrow$ $\sum_{m=1}^{N} \alpha_{m} c_{m}$ for all $N$, and we can let $N \rightarrow \infty$ to obtain

$$
\liminf _{n \rightarrow \infty} \frac{x_{n}}{n} \geq \kappa\left\langle c_{n}\right\rangle \text {. }
$$

To prove that also

$$
\liminf _{n \rightarrow \infty} \frac{x_{n}}{n} \leq \kappa\left\langle c_{n}\right\rangle
$$

we argue as follows. Set $c_{1}^{\prime}=0$, and $c_{n}^{\prime}=c_{n}$ for $n>1$. Then $x_{n}=n c_{1}+x_{n}^{\prime}$. Introducing

$$
G(z)=\sum_{n \geq 1} \frac{n^{n-1} x_{n}^{\prime}}{n !} z^{n}
$$

we have (see (4.12) and [9, equation (11.10)])

$$
G(z)=\frac{T(z)}{1-T(z)} \int_{0}^{z} \frac{C(w) d w}{w T(w)} .
$$

Since $1-T(z) \sim 2^{1 / 2}(1-e z)^{1 / 2}$ as $z \uparrow e^{-1}$, we have then

$$
\lim _{z \uparrow e^{-1}} 2^{1 / 2}(1-e z)^{1 / 2} G(z)=\int_{0}^{e^{-1}} \frac{C(w) d w}{w T(w)}=\kappa\left\langle c_{n}\right\rangle-c_{1} .
$$

By the Tauberian theorem for power series (see [2], for instance), we can write subsequently

$$
\sum_{j=1}^{n} \frac{j^{j-1} x_{j}^{\prime} e^{-j}}{j !}=(1+o(1))(2 \pi)^{-1 / 2} 2 n^{1 / 2}\left(\kappa\left\langle c_{n}\right\rangle-c_{1}\right) .
$$

According to Stirling's formula for factorials this leads to

$$
\liminf _{n \rightarrow \infty} \frac{x_{n}^{\prime}}{n} \leq \kappa\left\langle c_{n}\right\rangle-c_{1} \text {. }
$$


Given a sequence $c_{n}$ such that $\sum_{m \geq 1}\left|c_{m}\right| / m^{3 / 2}$ exists, we can define the residual sequence $r_{n}=x_{n}-n \kappa\left\langle c_{n}\right\rangle$. Residuals act linearly on $\left\langle c_{n}\right\rangle$, hence we can obtain further information about their asymptotic value by taking linear combinations of special sequences.

The residual of the constant sequence $\left\langle c_{n}\right\rangle$ with $c_{n}=1$ is -1 , because $x_{n}=2 n-1$. It follows from (4.6) that the residual of the sequence $\left\langle c_{n}(2 \beta)\right\rangle$ is $l(\beta) n^{1 / 2} c_{n}(2 \beta)+O\left(n^{\beta}\right)+O(1)$, when $\beta<\frac{1}{2}$. Therefore if $c_{n}=n^{\beta}$ we can put the estimates of Theorem 1 and Theorem 2 into a more precise form:

$$
x_{n}=n \kappa\left\langle n^{\beta}\right\rangle+l(\beta) n^{\beta+1 / 2}+O\left(n^{\beta}\right)+O(1), \quad \text { if } \beta<\frac{1}{2} .
$$

\section{ACKNOWLEDGMENT}

We wish to thank Philippe Flajolet for his penetrating remarks on a draft of this paper.

\section{REFERENCES}

1. Gotthold Eisenstein, Entwicklung von $\alpha^{\alpha^{a^{\circ}}}$, J. Reine Angew. Math. 28 (1844), 49-52.

2. William Feller, An introduction to probability theory and its applications, Vol. 2, Wiley, New York, 1971.

3. Philippe Flajolet and Andrew Odlyzko, Singularity analysis of generating functions, SIAM J. Discrete Math. (to appear).

4. Philippe Flajolet and Michèle Soria, Gaussian limiting distributions for the number of components in combinatorial structures, J. Combin. Theory Ser. A (to appear).

5. Donald E. Knuth, An analysis of optimum caching, J. Algorithms 6 (1985), 181-199.

6. _ The art of computer programming, Vol. 1: Fundamental algorithms, Addison-Wesley, Reading, Mass., 1973.

7. _ The art of computer programming, Vol. 2: Seminumerical algorithms, Addison-Wesley, Reading, Mass., 1981.

8. __ The art of computer programming, Vol. 3: Sorting and searching, Addison-Wesley, Reading, Mass., 1973.

9. Donald E. Knuth and Arnold Schönhage, The expected linearity of a simple equivalence algorithm, Theoret. Comput. Sci. 6 (1978), 281-315.

10. Martin Kruskal, The expected number of components under a random mapping function, Amer. Math. Monthly 61 (1954), 392-397.

11. Alfred Rényi, Some remarks on the theory of trees, Publ. Math. Inst. Hungar. Acad. Sci. 4 (1959), 73-85.

12. John Riordan, Combinatorial identities, Wiley, New York, 1968.

13. V. E. Stepanov, Predel' nye raspredelenila nekotorykh kharakteristik sluchainykh otobrazhenii, Teor. Verolatnost. Primenen. 14 (1969), 639-653; English transl., Limit distributions of certain characteristics of random mappings, Theor. Probab. Appl. 14 (1969), 612-626.

Department of Computer Science, Stanford University, Stanford, California 94305 (Current address of D. E. Knuth)

Current address (Boris Pittle): Department of Mathematics, Ohio State University, Columbus, Ohio 43210 